\title{
Regorafenib (BAY 73-4506) in advanced colorectal cancer: a phase I study
}

\section{Strumberg*,', ME Scheulen ${ }^{2}$, B Schultheis', H Richly ${ }^{2}$, A Frost ${ }^{3}$, M Büchert ${ }^{4}$, O Christensen $^{5}, M$ Jeffers $^{5}$, R Heinig ${ }^{6}, O$ Boix $^{6}$ and $K$ Mross $^{3}$}

'Department of Hematology and Medical Oncology, University of Bochum, Marienhospital Herne, Hölkeskampring 40, D-44625 Herne, Germany; ${ }^{2}$ West German Cancer Center, D-45 122 Essen, Germany; ${ }^{3}$ Tumor Biology Center, Albert-Ludwigs-University, Breisacherstr. 11 7, D-79 106 Freiburg, Germany; ${ }^{4}$ Magnetic Resonance Development and Application Center, University Medical Center, Breisacherstr. 60a, D-79I06 Freiburg, Germany; ${ }^{5}$ Bayer HealthCare Pharmaceuticals, Montville, NJ 07045-1000, USA; ${ }^{B}$ Bayer Pharma AG, D-42096 Wuppertal, Germany

BACKGROUND: In a phase I dose-escalation study, regorafenib demonstrated tolerability and antitumour activity in solid tumour patients. The study was expanded to focus on patients with metastatic colorectal cancer (CRC).

METHODS: Patients received oral regorafenib 60-220 mg daily ( $60 \mathrm{mg}$ daily in the extension cohort) in cycles of 21 days on, 7 days off treatment. Assessments included toxicity, response, pharmacokinetics and pharmacodynamics.

RESULTS: Thirty-eight patients with heavily pretreated CRC (median 4 prior lines of therapy, range 0-7) were enrolled in the dose-escalation and extension phases; 26 patients received regorafenib $160 \mathrm{mg}$ daily. Median treatment duration was 53 days (range 7-280 days). The most common treatment-related toxicities included hand-foot skin reaction, fatigue, voice change and rash. Twenty-seven patients were evaluable for response: I achieved partial response and 19 had stable disease. Median progression-free survival was 107 days $(95 \% \mathrm{Cl}, 66-161)$. At steady state, regorafenib and its active metabolites had similar systemic exposure. Pharmacodynamic assessment indicated decreased tumour perfusion in most patients.

CONCLUSION: Regorafenib showed tolerability and antitumour activity in patients with metastatic CRC. This expanded-cohort phase I study provided the foundation for further clinical trials of regorafenib in this patient population.

British Journal of Cancer (2012) I 06, 1722-1727. doi:I0.1038/bjc.2012.153 www.bjcancer.com

Published online 8 May 2012

(c) 2012 Cancer Research UK

Keywords: colorectal cancer; KRAS; regorafenib; tumour angiogenesis; multi-kinase inhibitor

Globally, colorectal cancer (CRC) is the fourth most common cancer in men and the third most common cancer in women (Parkin et al, 2005). Despite effective screening, 20-25\% of patients with CRC present with advanced, unresectable disease and are treated primarily with palliative systemic therapy (Hamilton and Grem, 1998).

5-Fluorouracil (5-FU)-based chemotherapy has long been the mainstay of systemic therapy for advanced or metastatic CRC (Cercek and Saltz, 2010). For first-line treatment, current guidelines recommend folinic acid +5 -FU + oxaliplatin (FOLFOX) or folinic acid +5 -FU + irinotecan (FOLFIRI) in combination with a targeted monoclonal antibody (bevacizumab, cetuximab or panitumumab; National Comprehensive Cancer Network, 2011). The use of the anti-epidermal growth factor receptor (EGFR) antibodies cetuximab and panitumumab is not recommended for patients whose tumours harbour KRAS mutations. There is no standard therapy after failure of all these therapies, and new effective treatment options are urgently needed.

Regorafenib (BAY 73-4506; Bayer Pharma AG, Berlin, Germany) is an orally active, potent multi-kinase inhibitor that targets a

\footnotetext{
*Correspondence: Dr D Strumberg;

E-mail: dirk.strumberg@marienhospital-herne.de

Received 8 December 2011; revised 29 February 2012; accepted 22

March 2012; published online 8 May 2012
}

broad range of angiogenic, stromal and oncogenic kinases, including vascular endothelial growth factor (VEGF) receptors 1, 2 and 3, tyrosine kinase with immunoglobulin and epidermal growth factor homology domain 2 (TIE-2), platelet-derived growth factor receptor- $\beta$, c-kit, ret, raf- 1 and BRAF (both wildtype and the V600E mutant; Wilhelm et al, 2011). In preclinical studies, regorafenib demonstrated antitumour activity in a broad spectrum of xenograft models, including CRC (Wilhelm et al, 2011).

A first-in-human, open-label, non-randomised, phase I doseescalation study in patients with advanced solid tumours demonstrated that single-agent regorafenib was well tolerated up to the recommended phase II dose of $160 \mathrm{mg}$ once daily, given in cycles of 21 days on, 7 days off. Preliminary activity was observed in patients with CRC (Mross et al, 2012). This study was expanded to further evaluate regorafenib ( $160 \mathrm{mg}$ once daily) in patients with advanced or metastatic CRC.

\section{PATIENTS AND METHODS}

The study took place at three specialist cancer centres in Germany between July 2005 and June 2009. The protocol was approved by the independent ethics committee at each study centre. Before enrolment, all patients had to provide written, informed consent to participate. The study met all local legal and regulatory requirements and followed the Declaration of Helsinki. 


\section{Patients}

In the dose-escalation phase of the study, patients with advanced, histologically or cytologically confirmed solid tumours (including CRC), non-Hodgkin's lymphoma or multiple myeloma were eligible. An additional extension cohort was planned, to focus on patients with cancer types that were considered to be promising targets for regorafenib, based on experience gained in the doseescalation phase. In the event, CRC was identified as the tumour type of most interest for further analysis; thus the extension phase included patients with advanced, histologically confirmed CRC.

Patients had to be aged at least 18 years and have an Eastern Cooperative Oncology Group performance status of 0-2, life expectancy of at least 12 weeks, and adequate bone marrow, liver and renal function. Key exclusion criteria included a history of cardiac disease, congestive heart failure (New York Heart Association classes II-IV), uncontrolled hypertension, human immunodeficiency virus infection, active hepatitis $\mathrm{B}$ or $\mathrm{C}$ virus infection or other active clinically serious infections (grade $>2$ according to the National Cancer Institute Common Terminology Criteria for Adverse Events (NCI CTC-AE) v3.0).

The participants had to have malignancies that were refractory to standard treatment or for which no standard therapy was available; in addition, patients could be included if a standard treatment was available but the patient had refused it. During the study, patients did not receive any other anticancer therapy.

\section{Dosing and administration}

In the initial dose-escalation phase, patients received once daily oral doses of regorafenib ranging from 60 to $220 \mathrm{mg}$ in repeating 28-day cycles of 21 days on treatment, 7 days off treatment. In the extension cohort, patients received regorafenib $160 \mathrm{mg}$ orally once daily ( 21 days on, 7 days off treatment).

Treatment continued until tumour progression, occurrence of unacceptable toxicity or withdrawal of consent. Interruptions in dosing or dose reductions were permitted if a patient experienced an adverse event that was considered to be related to the study medication and was at least grade 3 in severity, according to NCI CTC-AE v3.0. Individual patients could not have their dose increased.

\section{Assessments}

A pre-study examination was performed within 14 days before the first treatment dose. A complete end-of-treatment assessment was performed within 14 days of ending regorafenib treatment. Other assessments were undertaken as described below.

Safety evaluations included physical examinations, electrocardiogram, standard laboratory tests and measurement of vital signs. Adverse events were assessed according to NCI CTC-AE v3.0. Patients were reviewed for evidence of cumulative toxicity from repeated cycles of treatment. A follow-up visit was made $30 \pm 4$ days after the last administration of regorafenib to collect information on ongoing toxicities or significant adverse events. Any adverse events that occurred within 30 days of the last dose of study medication were followed until resolution.

Tumour response and progression were evaluated using Response Evaluation Criteria in Solid Tumors (RECIST) v1.1 (Eisenhauer et al, 2009). All lesions were measured using X-ray, magnetic resonance imaging (MRI) or computed tomography at baseline, and were assessed and reported at the end of every second treatment cycle beginning at baseline, unless progression was noted.

Progression-free survival (PFS) was measured as the number of days from start of treatment to progression, death before progression (patient not censored) or last tumour evaluation at which the patient was known to have not progressed (patient censored). The Kaplan-Meier product-limit estimate of PFS was derived for all patients and for those with KRAS-mutated or wild-type tumours.

KRAS mutations were analysed using archival tumour or plasma samples. Using the QIAamp DNA Micro Kit (Qiagen, Crawley, UK), DNA was isolated from formalin-fixed, paraffin-embedded tissue sections of archival tumour biopsies collected at diagnosis, and mutational analysis was performed by Sequenom, Inc. (San Diego, CA, USA) using OncoCarta v1.0, which examines 238 mutational 'hotspots' in 19 different cancer-related genes. Plasma samples collected at baseline or during the study were analysed for mutations using the BEAMing technology (Inostics $\mathrm{GmbH}$, Hamburg, Germany; Dressman et al, 2003).

Plasma concentrations of regorafenib and its pharmacologically active metabolites M2 ( $N$-oxide metabolite; BAY 75-7495) and M5 ( $N$-oxide/ $N$-desmethyl metabolite; BAY 81-8752) were measured in blood samples collected on days 1 and 21 of cycle 1 (doseescalation cohort) and day 21 of cycles 1 and 2 (extension cohort). Analyte concentrations were determined by high-performance liquid chromatography with tandem mass spectrometric detection, with a lower quantification limit of $2 \mu \mathrm{gl}^{-1}$. Analyses were performed in accordance with the Food and Drug Administration guidelines on bioanalytical validation (Food and Drug Administration, 2001). Pharmacokinetic parameters were calculated using standard non-compartmental methods (WinNonlin Version 4.1; Pharsight Corporation, Mountain View, CA, USA) and summarised as geometric mean and geometric coefficient of variation (CV). Point estimators (least square means) and twosided $90 \%$ confidence intervals for area under the concentrationtime curve from 0 to $24 \mathrm{~h}\left(\mathrm{AUC}_{0-24}\right)$ and maximum concentration $\left(C_{\max }\right)$ ratios (cycle 2:cycle 1 in extension cohort) were calculated using an analysis of variance model.

Tumour-perfusion properties were measured using dynamic contrast-enhanced (DCE) MRI at screening, on days 2 and 21 of cycle 1 , on day 21 of cycles $2-4$, on day 21 of every second cycle thereafter, and at the final visit. The area under the contrast agent concentration-time curve during the first $60 \mathrm{~s}$ after arrival of the contrast agent $\left(\mathrm{iAUC}_{60}\right.$ ) was used as the DCE-MRI endpoint (Mross et al, 2009). The association between DCE-MRI endpoint and PFS was investigated by censored data linear rank statistics based on the Wilcoxon scores using the DCE-MRI endpoint as covariate.

\section{RESULTS}

Results are presented for all 38 patients with metastatic CRC who were enrolled in this phase I study during the dose-escalation $(n=15)$ and extension $(n=23)$ phases. Patient demographic and baseline characteristics are shown in Table 1. Patients had previously received a median of 4 lines (range $0-7$ ) of systemic therapy for metastatic disease, including oxaliplatin, irinotecan, bevacizumab and anti-EGFR antibodies; one patient with no prior treatment had refused to receive any systemic treatment before enrolling in this study. Twenty-eight patients had received regimens containing both oxaliplatin and irinotecan.

Table 2 shows the number of patients receiving regorafenib at each dose level and the duration of treatment. Reasons for discontinuation of regorafenib were progressive disease $(n=19$; $50 \%)$, consent withdrawal $(n=5 ; 13 \%)$, treatment-related adverse events $(n=11 ; 29 \%)$ and non-treatment-related adverse events $(n=3 ; 8 \%)$.

\section{Safety}

Treatment-emergent, drug-related adverse events were reported in $32(84 \%)$ of the 38 patients with CRC (Table 3). All treatment- 
Table I Patient demographics and baseline disease characteristics

\begin{tabular}{lc}
\hline Characteristics & Patients (n=38) \\
\hline Sex, $n$ (\%) & $21(55)$ \\
Male & $17(45)$ \\
Female & $64(36-85)$ \\
Median (range) age, years & \\
Ethnicity, $n$ (\%) & $37(97)$ \\
Caucasian & $1(3)$ \\
Asian & \\
ECOG performance status, $n$ (\%) & $18(47)$ \\
0 & $18(47)$ \\
I & $2(5)$ \\
2 & \\
Previous treatment, $n$ (\%) & $38(100)$ \\
Surgery & $11(29)$ \\
Radiotherapy & $37(97)$ \\
Systemic therapy & $32(84)$ \\
Oxaliplatin & $32(84)$ \\
Irinotecan & $20(53)$ \\
Bevacizumab & $20(53)$ \\
Anti-EGFR antibody (cetuximab or panitumumab) & \\
Median previous chemotherapy regimens, $n$ (range) & $4(0-7)$ \\
Metastatic sites at screening, $n$ (\%) & \\
I-2 & \\
$\geqslant 3$ & $26(68)$ \\
\hline
\end{tabular}

Abbreviations: ECOG = Eastern Cooperative Oncology Group; EGFR = epidermal growth factor receptor.

Table 2 Dose levels and treatment duration of regorafenib

\begin{tabular}{|c|c|c|c|c|c|}
\hline \multirow{2}{*}{$\begin{array}{l}\text { Regorafenib } \\
\text { administration }\end{array}$} & \multicolumn{5}{|c|}{ Dose level } \\
\hline & $60 \mathrm{mg}^{\mathrm{a}}$ & $120 \mathrm{mg}^{\mathrm{a}}$ & $160 \mathrm{mg}^{\mathrm{b}}$ & $220 \mathrm{mg}^{\mathrm{b}}$ & Overall \\
\hline $\begin{array}{l}\text { Colorectal cancer } \\
\text { patients, } n\end{array}$ & I & 4 & 26 & 7 & 38 \\
\hline $\begin{array}{l}\text { Median (range) treatment } \\
\text { duration, days }\end{array}$ & 49 & $\begin{array}{c}46 \\
(7-129)\end{array}$ & $\begin{array}{c}49 \\
(8-280)\end{array}$ & $\begin{array}{c}106 \\
(56-219)\end{array}$ & $\begin{array}{c}53 \\
(7-280)\end{array}$ \\
\hline
\end{tabular}

a Patients at 60 and $120 \mathrm{mg}$ received regorafenib as solution. ${ }^{\mathrm{b}}$ Patients at 160 and $220 \mathrm{mg}$ received regorafenib as tablets with a bioavailability of $70 \%$ (20 $\mathrm{mg}$ tablet) to $83 \%$ ( $100 \mathrm{mg}$ tablet) of the solution.

related adverse events were grade 3 or lower, except one case of clinically asymptomatic grade 4 thrombocytopenia. No grade 5 adverse events were reported. Dose reduction or interruption of regorafenib was necessary in 25 patients $(66 \%)$. The most common adverse event leading to dose reduction was hand-foot skin reaction. Overall, regorafenib was permanently discontinued in 11 patients $(29 \%)$ due to a treatment-related adverse event. Among the 25 patients treated at the $160 \mathrm{mg}$ dose level, 6 patients permanently discontinued regorafenib due to treatment-related adverse events (hand-foot skin reaction $n=1$; hypertension $n=1$; fatigue $n=1$; fatigue and other constitutional symptoms $n=1$; thrombocytopenia $n=1$; duodenal ulcer $n=1$ ).

\section{Efficacy}

Eleven patients were not evaluable for tumour response for the following reasons: discontinuation of treatment due to adverse
Table 3 Treatment-emergent drug-related adverse events affecting at least $10 \%$ of patients at any grade or resulting in treatment discontinuation

\begin{tabular}{|c|c|c|}
\hline & \multicolumn{2}{|c|}{$\begin{array}{l}\text { Patients experiencing } \\
\text { an adverse event, } n \text { (\%) }\end{array}$} \\
\hline & All grades & Grade $\geqslant 3^{a}$ \\
\hline All events & $34(84)$ & $22(58)$ \\
\hline \multicolumn{3}{|l|}{ By CTC-AE category } \\
\hline Dermatological adverse events & $26(68)$ & $13(34)$ \\
\hline Hand-foot skin reaction & $23(6 \mid)$ & $12(32)$ \\
\hline Dry skin & $7(18)$ & 0 \\
\hline Rash, desquamation & | | (29) & $2(5)$ \\
\hline Alopecia & $4(11)$ & 0 \\
\hline Fatigue & $19(50)$ & $4(11)$ \\
\hline Voice changes & $13(34)$ & I (3) \\
\hline Anorexia & $9(24)$ & 0 \\
\hline Diarrhoea & $9(24)$ & I (3) \\
\hline Hypertension & $7(18)$ & $4(11)$ \\
\hline Oral mucositis & $7(18)$ & 0 \\
\hline Pain (muscle) & $7(18)$ & 0 \\
\hline Dry mouth & $6(16)$ & I (3) \\
\hline Weight loss & $5(13)$ & 0 \\
\hline Auditory, ear & $4(11)$ & 0 \\
\hline Thrombocytopenia & $4(11)$ & I (3) \\
\hline
\end{tabular}

Abbreviation: CTC-AE = Common Terminology Criteria Adverse Event. ${ }^{a}$ All grade 3, except one patient with grade 4 thrombocytopenia. ${ }^{b}$ Any event in National Cancer Institute Common Terminology CTC-AE class.

events before the first tumour assessment at the end of cycle 2 $(n=8)$; consent withdrawal before the tumour assessment at the end of cycle $2(n=1)$; diagnosis of brain metastasis 1 day after the start of treatment $(n=1)$; and missing tumour assessment $(n=1)$. As a result, 27 patients were evaluable for response. Best responses included a confirmed partial response in one patient $(4 \%)$ and stable disease in 19 patients (70\%), giving a disease control rate (partial response or stable disease lasting for at least 2 months) of $74 \%$. Seven patients $(26 \%)$ had progressive disease as best response. Patients achieving partial response or stable disease had received previous treatment with cetuximab or panitumumab $(45 \%)$, bevacizumab $(45 \%)$, oxaliplatin $(85 \%)$ or irinotecan $(80 \%)$. The maximum percentage change in tumour size from baseline for each patient is shown in Figure 1. Tumour shrinkage (of any percentage at any time during the study) was observed in 13 patients.

Median PFS was 107 days (95\% CI, 66-161). At data cutoff, 13 patients had PFS of $>100$ days (Figure 2).

\section{KRAS mutation analysis}

KRAS mutations were found in 19 of 37 patients with tumour or plasma samples; 18 patients had wild-type KRAS and 1 had no available sample (see Figure 2). The Kaplan-Meier analysis shows no clear difference in PFS between KRAS wild-type and mutant group, even though median PFS was 84 days in the mutant group and 161 days in the wild-type group. The data from this exploratory mutational analysis performed in this relatively small number of subjects with a relatively high number of censored data indicate that the presence of a KRAS mutation does not preclude regorafenib activity.

\section{Pharmacokinetic assessments}

Plasma concentration-time profiles at steady state (day 21 of cycles 1 and 2) of regorafenib and its metabolites M2 and M5 are 


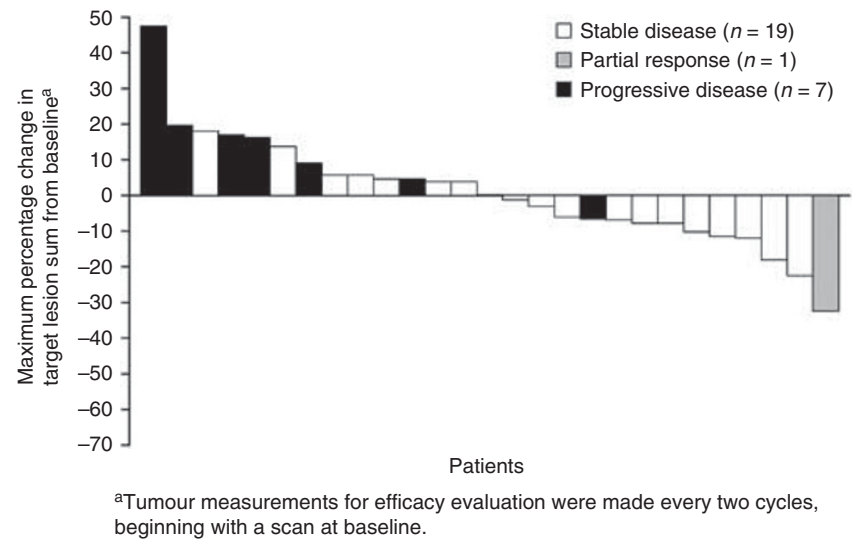

Figure I Maximum percentage change in target lesion sum from baseline $(n=27)$.

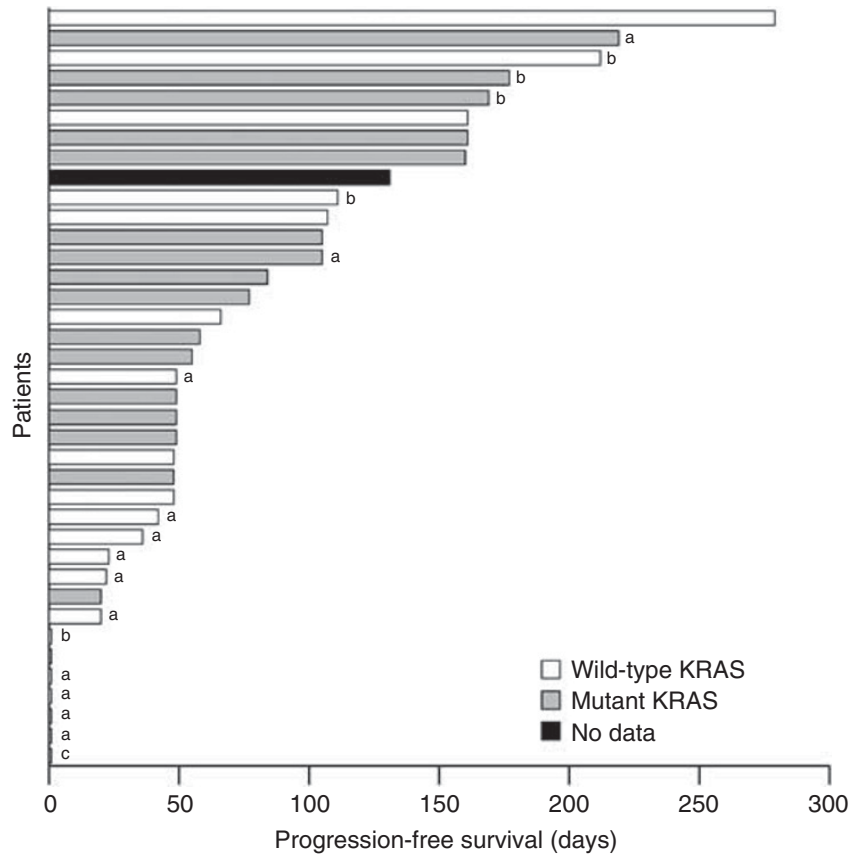

aData censored; patients dropped out due to an adverse event; presented is the time from start of treatment to last tumour assessment showing stable disease.

bData censored; patients dropped out due to consent withdrawn; presented is the time from start of treatment to last tumour assessment showing stable disease.

'Data censored; patient dropped out 1 day after start of treatment due to diagnosis of brain metastases.

Figure 2 Progression-free survival for individual patients, showing KRAS mutational status.

shown in Figure 3. The difference between $C_{\max }$ and minimum concentration $\left(C_{\mathrm{min}}\right)$ within the dosing interval of $24 \mathrm{~h}$ was small, with mean $C_{\max }: C_{\min }$ ratios of $\sim 2-3$ for both regorafenib and $\mathrm{M} 2$, and $\sim 1.5$ for M5. Steady-state pharmacokinetic profiles demonstrated similar mean systemic exposure of regorafenib and its metabolites. Ratios for cycle 2:cycle 1 are shown in Table 4. $\mathrm{AUC}_{0-24}$ and $C_{\max }$ at steady state showed pronounced variability between patients, with inter-individual CV of $\sim 60-90 \%$ for regorafenib and $\mathrm{M} 2$, and $>100 \%$ for $\mathrm{M} 5$. The intra-individual CVs for $C_{\max }$ and $\mathrm{AUC}_{0-24}(n=14)$ were $32-34 \%$ for regorafenib, $37-46 \%$ for M2 and $52-63 \%$ for M5 (see Table 4). Intra-individual variability was therefore markedly reduced compared with interindividual variability. At the $160 \mathrm{mg}$ dose, plasma exposure at steady state $\left(\mathrm{AUC}_{0-24}\right.$, geometric mean) of the metabolites $\mathrm{M} 2$ (48 $\left.\mathrm{mg} \mathrm{h}^{-1}\right)$ and M5 (65-79 $\mathrm{mg} \mathrm{h}^{-1}$ ) was similar to, or slightly

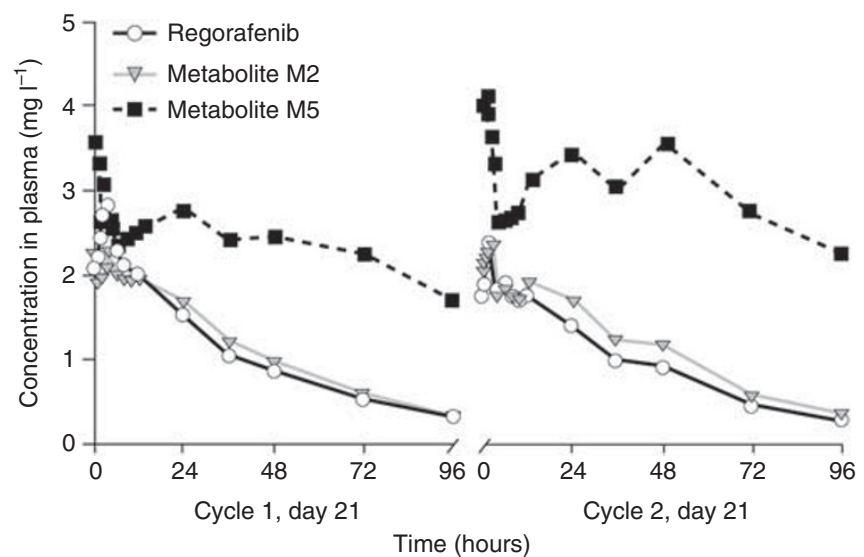

Figure 3 Concentration-time profile of regorafenib and its metabolites M2 (N-oxide metabolite) and M5 (N-oxide/N-desmethyl metabolite).

greater than, that of regorafenib $\left(45-50 \mathrm{mgh}^{-1}\right)$. The terminal half-life of M2 $(25 \mathrm{~h})$ was comparable to that of regorafenib $(26-28 \mathrm{~h})$, although the elimination of M5 was slower, with an estimated half-life of 51-64h.

\section{Pharmacodynamic assessments}

Tumour perfusion in representative lesions was measured by DCEMRI and the AAUC $_{60}$ data are shown in Figure 4. The median ratio to baseline was 0.803 (range $0.367-1.799, n=26$ ) on day 2 of cycle $1,0.661$ (range $0.129-1.143, n=22$ ) on day 21 of cycle 1 and 0.507 (range $0.031-1.53, n=20$ ) on day 21 of cycle 2 . There was no apparent association between iAUC $_{60}$ (natural logarithm of ratio to baseline) and PFS $(P=0.74)$.

\section{DISCUSSION}

The current study evaluated regorafenib in 38 patients with heavily pretreated, advanced or metastatic CRC. Regorafenib was well tolerated in this study. The most common treatment-related adverse events included hand-foot skin reactions, fatigue, voice changes, anorexia and diarrhoea. In general, adverse events were manageable in an outpatient setting with dose reduction or interruption. These findings are consistent with the safety profile observed in patients with other solid tumours (Mross et al, 2012) and with results from a phase II study of regorafenib in patients with untreated metastatic or unresectable renal cell carcinoma (Eisen et al, 2011).

In this study, regorafenib demonstrated clinical efficacy in patients with metastatic CRC. According to RECIST, a disease control rate of $74 \%$ was achieved ( $4 \%$ partial response, $70 \%$ stable disease). Such activity is encouraging, given that, with one exception (a patient who had refused chemotherapy), these patients had been heavily pretreated: the vast majority of patients had failed standard therapies containing 5-FU, oxaliplatin and irinotecan; approximately half of the patients had also received either anti-VEGF or anti-EGFR agents (including some patients who appear to have received anti-EGFR therapy despite having KRAS-mutant tumours-possibly at a time when the importance of KRAS status was not as universally understood as it is now). Best supportive care or participation in a clinical trial is usually recommended in this setting (Cercek and Saltz, 2010; National Comprehensive Cancer Network, 2011). Although only one confirmed partial response was observed in this study, most patients achieved disease stabilisation, which has been shown to be clinically meaningful (Akbulut et al, 2004; Bitossi et al, 2008; van 


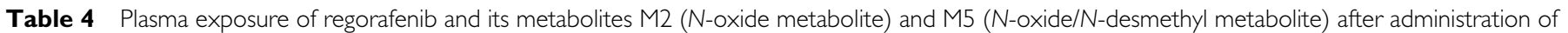
$160 \mathrm{mg}$ tablet daily for 21 days

\begin{tabular}{|c|c|c|c|c|c|}
\hline & & $\begin{array}{c}\text { Cycle I, day } 2 \text { I, geometric } \\
\text { mean }(\% \mathrm{CV} ; n=19)\end{array}$ & $\begin{array}{c}\text { Cycle 2, day } 21 \text {, geometric } \\
\text { mean }(\% \mathrm{CV} ; n=14)\end{array}$ & $\begin{array}{c}\text { Intra-individual } \\
\text { variability }(\% \mathrm{CV} ; n=14)\end{array}$ & $\begin{array}{c}\text { Cycle 2:cycle I ratio } \\
\text { (two-sided }\left(90 \% \mathrm{Cls}^{\mathrm{a}} ; n=14\right)\end{array}$ \\
\hline Regorafenib & $\begin{array}{l}\mathrm{AUC}_{0-24}, \mathrm{mghl}^{-1} \\
\mathrm{C}_{\max },\left.\mathrm{mg}\right|^{-1}\end{array}$ & $\begin{array}{l}50.26(85.5) \\
3.450(62.8)\end{array}$ & $\begin{array}{l}45.22(87.9) \\
3.229(72.6)\end{array}$ & $\begin{array}{l}34.22 \\
32.41\end{array}$ & $\begin{array}{l}91.61(73.32-114.47) \\
94.11(76.17-116.29)\end{array}$ \\
\hline$M 2$ & $\begin{array}{l}\mathrm{AUC}_{0-24}, \mathrm{mghl}^{-1} \\
\mathrm{C}_{\max },\left.\mathrm{mg}\right|^{-1}\end{array}$ & $\begin{array}{l}48.08(88.5) \\
3.171(72.4)\end{array}$ & $\begin{array}{l}47.74(77.8) \\
3.269(61.9)\end{array}$ & $\begin{array}{l}45.94 \\
37.19\end{array}$ & $\begin{array}{l}102.84(76.72-137.83) \\
105.2 \mid(82.68-133.86)\end{array}$ \\
\hline M5 & $\begin{array}{l}\mathrm{AUC}_{0-24}, \mathrm{mghl}^{-1} \\
\mathrm{C}_{\max },\left.\mathrm{mg}\right|^{-1}\end{array}$ & $\begin{array}{l}64.58(182.4) \\
3.994(173.5)\end{array}$ & $\begin{array}{l}79.44(138.7) \\
5.145(141.0)\end{array}$ & $\begin{array}{l}63.48 \\
51.56\end{array}$ & $\begin{array}{l}\mid 24.66(84.45-184.03) \\
\mid 32.33(95.6|-| 83.14)\end{array}$ \\
\hline
\end{tabular}

Abbreviations: $A \cup C(0-24)=$ area under the concentration-time curve from 0 to $24 \mathrm{~h} ; \mathrm{Cl}=$ confidence interval; $C_{\text {max }}=$ maximum concentration; $\mathrm{CV}=$ coefficient of variation. apoint estimators (least square means).

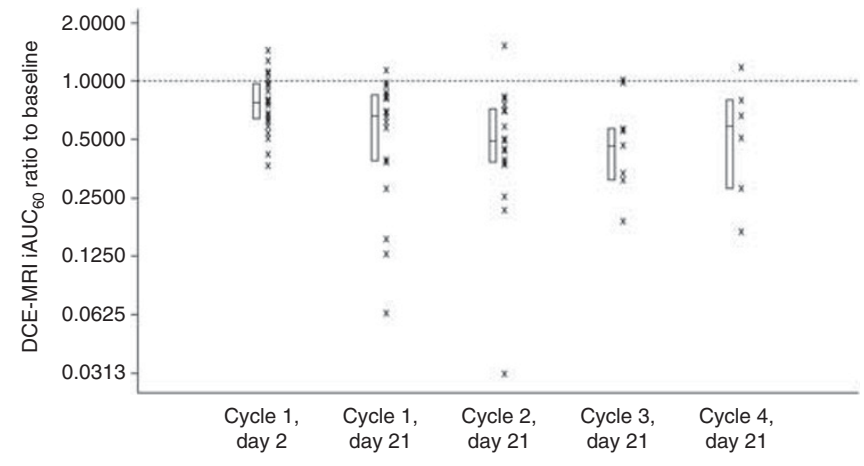

Boxplot displays median, $25 \%$, and $75 \%$ percentiles.

Only patients with valid biomarker data $\left(\mathrm{iUC}_{60}\right)$ at baseline and during treatment are displayed $(n=28)$

Figure 4 Dynamic contrast-enhanced magnetic resonance imaging: ratio to baseline of the area under the contrast agent concentration-time curve during the first $60 \mathrm{~s}$ after arrival of the contrast agent.

Cutsem et al, 2008). One group estimated that in patients with chemorefractory metastatic CRC receiving panitumumab $\sim 80 \%$ of the treatment effect on PFS was due to disease stabilisation (van Cutsem et al, 2007). Therefore, it is possible that the high disease control rate observed with regorafenib may translate into a survival benefit. However, it must be borne in mind that this was primarily a dose-finding and safety study and the true survival impact needs to be verified in large randomised controlled trials.

Angiogenesis is a validated therapeutic target for CRC. Bevacizumab, an anti-VEGF-A antibody, in combination with standard chemotherapy improves PFS and overall survival compared with chemotherapy alone in metastatic CRC (Hurwitz et al, 2004); to date, bevacizumab is the only antiangiogenic agent approved for the treatment of CRC. Consistent with preclinical data, pharmacodynamic assessment by DCE-MRI in the present phase I study indicated reduced tumour perfusion after regorafenib treatment in a majority of patients with metastatic CRC, suggesting that regorafenib may have antiangiogenic effects. However, DCE-MRI has not been fully validated as a pharmacodynamic marker for antiangiogenic agents. In this study, no apparent correlation was observed between DCE-MRI change and PFS. Given the broad kinase inhibitory spectrum of regorafenib, inhibition of other, non-angiogenic kinases may have contributed to the reported clinical activity. Further investigations are required to fully elucidate the mechanism of action of regorafenib (angiogenic and non-angiogenic) in CRC.

Mutation in the KRAS gene is a well established biomarker in CRC that clearly predicts lack of activity with the anti-EGFR agents cetuximab and panitumumab (Cercek and Saltz, 2010; National Comprehensive Cancer Network, 2011). An exploratory mutational analysis was undertaken in the present study to investigate whether KRAS status might have an impact on response to regorafenib. Kaplan-Meier analysis showed no statistically significant difference in PFS between patients with KRAS wild-type and mutant tumours. However, the small number of patients (with a relatively high number of censored data) resulted in wide confidence intervals. Thus, no firm conclusions can be drawn about the impact of KRAS status on response to regorafenib therapy from the present study. Further investigations in larger patient populations are needed to provide more conclusive answers to this question.

Systemic exposures of regorafenib and its active metabolites M2 and M5 at steady state demonstrated markedly lower intra-individual than inter-individual variability. Compared with the parent drug, M2 and M5 had similar or slightly greater systemic exposure, and similar or slower elimination. In preclinical in vitro and xenograft studies, M2 and M5 have been shown to be pharmacologically active, with efficacies similar to the parent compound (Zopf et al, 2010). Therefore, the M2 and M5 metabolites of regorafenib are likely to contribute to clinical activity.

In conclusion, regorafenib $160 \mathrm{mg}$ orally once daily in cycles of 21 days on, 7 days off treatment was tolerable, had a manageable adverse event profile and demonstrated clinical activity in heavily pretreated patients with metastatic CRC. As a result of the outcomes from this study, it was deemed appropriate to consider $\mathrm{CRC}$ as a focus for further investigation of regorafenib, including a randomised, double-blind, placebo-controlled, phase III study of best supportive care plus either regorafenib or placebo in patients with metastatic CRC, who have progressed after standard therapy (the CORRECT study; ClinicalTrials.gov identifier: NCT01103323).

\section{ACKNOWLEDGEMENTS}

We thank Dr Frank-Thorsten Hafner for the bioanalytical work on regorafenib and metabolites, as well as Katherine Wilson and Sara Black for writing assistance in the preparation and revision of the draft manuscript. Bayer Pharma AG (Berlin, Germany) sponsored this study and provided funding for medical writing assistance.

\section{REFERENCES}

Akbulut H, Icli F, Yalcin B, Demirkazik A, Onur H, Buyukcelik A, Utkan G (2004) Activity of irinotecan, cisplatin and dacarbazine (CPD) combination in previously treated patients with advanced colorectal carcinoma. Exp Oncol 26: 149-152

Bitossi R, Sculli CM, Tampellini M, Alabiso I, Brizzi MP, Ferrero A, Ottone A, Bellini E, Gorzegno G, Berruti A, Dogliotti L (2008) Gemcitabine and protracted 5-fluorouracil infusion as third-line chemotherapy in refractory colorectal cancer patients. Anticancer Res 28: 3055-3060 
Cercek A, Saltz L (2010) Evolving treatment of advanced colorectal cancer. Curr Oncol Rep 12: 153-159

Dressman D, Yan H, Traverso G, Kinzler KW, Vogelstein B (2003) Transforming single DNA molecules into fluorescent magnetic particles for detection and enumeration of genetic variations. Proc Natl Acad Sci USA 100: 8817-8822

Eisen T, Joensuu H, Nathan P, Harper P, Wojtukiewicz M, Nicholson S, Bahl B, Tomczak P, Wagner A, Quinn D (2011) Phase II trial of the oral multikinase inhibitor regorafenib (BAY 73-4506) as first-line therapy in patients with metastatic or unresectable renal cell cancer (RCC) Eur J Cancer 47(Suppl): Abstract 7. 141

Eisenhauer EA, Therasse P, Bogaerts J, Schwartz LH, Sargent D, Ford R, Dancey J, Arbuck S, Gwyther S, Mooney M, Rubinstein L, Shankar L, Dodd L, Kaplan R, Lacombe D, Verweij J (2009) New response evaluation criteria in solid tumours: revised RECIST guideline (version 1.1). Eur J Cancer 45: 228-247

Food and Drug Administration (2001) Guidance for Industry. Bioanalytical Method Validation. Food and Drug Administration: Silver Spring, MD, USA

Hamilton J, Grem J (1998) Lower gastrointestinal tract cancer. In Current Cancer Therapeutics, Kirkwood JM, Lotze MT, Yasko JM (eds) 3rd edn, pp 156-160. Churchill Livingstone, Current Medicine Inc.: Philadelphia, PA, USA

Hurwitz H, Fehrenbacher L, Novotny W, Cartwright T, Hainsworth J, Heim W, Berlin J, Baron A, Griffing S, Holmgren E, Ferrara N, Fyfe G, Rogers B, Ross R, Kabbinavar F (2004) Bevacizumab plus irinotecan, fluorouracil, and leucovorin for metastatic colorectal cancer. $N$ Engl J Med 350: 2335-2342

Mross K, Fasol U, Frost A, Benkelmann R, Kuhlmann J, Büchert M, Unger C, Blum H, Hennig J, Milenkova TP, Tessier J, Krebs AD, Ryan AJ, Fischer R (2009) DCE-MRI assessment of the effect of vandetanib on tumor vasculature in patients with advanced colorectal cancer and liver metastases: a randomized phase I study. J Angiogenes Res 1: 5
Mross K, Frost A, Steinbild S, Hedborn S, Büchert M, Fasol U, Unger C, Kraetzschmar J, Heinig R, Boix O, Christensen O (2012) A phase I dose escalation study of regorafenib (BAY 73-4506), an inhibitor of oncogenic, angiogenic and stromal kinases, in patients with advanced solid tumors Clin Cancer Res; e-pub ahead of print 20 April 2012

National Comprehensive Cancer Network (2011) NCCN Clinical Practice Guidelines in Oncology. National Comprehensive Cancer Network: Fort Washington, PA, USA

Parkin DM, Bray F, Ferlay J, Pisani P (2005) Global cancer statistics, 2002. CA Cancer J Clin 55: 74-108

Van Cutsem E, Siena S, Humblet Y, Canon JL, Maurel J, Bajetta E, Neyns B, Kotasek D, Santoro A, Scheithauer W, Spadafora S, Amado RG, Hogan N, Peeters M (2008) An open-label, single-arm study assessing safety and efficacy of panitumumab in patients with metastatic colorectal cancer refractory to standard chemotherapy. Ann Oncol 19: 92-98

Van Cutsem E, Peeters M, Siena S, Humblet Y, Hendlisz A, Neyns B, Canon JL, Van Laethem JL, Maurel J, Richardson G, Wolf M, Amado RG (2007) Open-label phase III trial of panitumumab plus best supportive care compared with best supportive care alone in patients with chemotherapy-refractory metastatic colorectal cancer. J Clin Oncol 25: 1658-1664

Wilhelm SM, Dumas J, Adnane L, Lynch M, Carter CA, Schütz G, Thierauch KH, Zopf D (2011) Regorafenib (BAY 73-4506): a new oral multikinase inhibitor of angiogenic, stromal and oncogenic receptor tyrosine kinases with potent preclinical antitumor activity. Int J Cancer 129: 245-255

Zopf D, Heinig R, Thierauch K-H, Hirth-Dietrich C, Hafner F-T, Christensen O, Lin T, Wilhelm S, Radtke M (2010) Regorafenib (BAY 73-4506): preclinical pharmacology and clinical identification and quantification of its major metabolites (17-21 April) (2010) At the American Association for Cancer Research 101st Annual Meeting, Washington, DC, USA

This work is published under the standard license to publish agreement. After 12 months the work will become freely available and the license terms will switch to a Creative Commons Attribution-NonCommercial-Share Alike 3.0 Unported License. 